\section{Outpatient treatment of venous diseases with medical compression stockings in Germany}

\author{
Christine Schwahn-Schreiber, ${ }^{1}$ \\ Markward Marshall, ${ }^{2}$ Renate Murena- \\ Schmidt,${ }^{3}$ Wiebke Doppel ${ }^{4}$ \\ ${ }^{1}$ Institute of Vascular Medicine, \\ Otterndorf, Germany; ${ }^{2}$ Institute of \\ Vascular Medicine, Tegernsee, \\ Germany; ${ }^{3}$ Center for Vascular Diseases, \\ Cologne, Germany, ${ }^{4}$ medi GmbH \& Co. \\ KG, Bayreuth, Germany
}

\begin{abstract}
The aim of this study was to assess the provision of medical compression stockings (MCS) to venous outpatients in daily practice, the tolerability of mediven-MCS, and the compliance and subjective and objective symptoms of patients. During the 18-month observational study in Germany, 531 venous outpatients were evaluated regarding MCSprescriptions and demographic, physical, and clinical findings. We found that MCS were mostly of compression class 2 and light textile characteristics, irrespective of the patients' CEAP and Body Mass Index (BMI). During the study, fewer prescriptions for MCS and donning aids were dispensed than would have been possible according to patient's needs and health insurance regulations. Findings suggesting impaired MCStolerability rarely occurred. Prevention of constrictions, compliance of patients, and CEAP-class (C3-patients) could be improved when MCS and donning aids were individually prescribed according to the patient's individual CEAP, BMI, and age. In conclusion, patient- and disease-specific characteristics of venous patients require a more individual consideration regarding number and type of MCS-prescriptions.
\end{abstract}

\section{Introduction}

Worldwide, treatment with medical compression stockings (MCS) is a central element of the therapy and prevention of venous diseases and impairment of venous function, respectively. ${ }^{1-3}$ Several studies have demonstrated MCS effectiveness in the treatment ${ }^{4-6}$ and prevention ${ }^{7,8}$ of venous disorders, in postoperative care, $, 9,10$ and in prevention of recurrence. ${ }^{11,12}$

To be successful, MCS therapy requires careful consideration of disease- and patient-specific factors including CEAP class, leg circumference, edema, age, and body mass index (BMI). ${ }^{13}$ Appropriate MCS parameters such as compression class (CCL), knitting variety (round-/flat-knitted), stocking length, and MCS characteristics (characteristics comprises textile MCS features affecting elasticity and other MCS characteristics) need to be set individually for every patient. In addition, outcome of MCS treatment is affected by the patient's compliance, which in turn is potentially influenced by some of the above-mentioned factors. ${ }^{14,15}$ Guidelines such as the German MCS guideline of $2006^{3}$ might give suggestions as to how to use MCS in different indications, but they usually do not assign definite MCS parameters to specific venous diseases, symptoms, and patient characteristics. Moreover, little is known on the provision of MCS to venous outpatients in daily practice in Germany, ${ }^{16}$ i.e. if MCS prescriptions adequately differentiate between CCL, knitting variety, length, and characteristics, if MCS are well tolerated, and how tolerability is affected by both MCS and patient characteristics. Here, we report the results of a large multicenter observational study ${ }^{17,18}$ on the outpatient medical care situation in Germany. The data evaluated included provision of MCS and donning aids to 531 venous outpatients, safety and tolerability of mediven round-knitted MCS, as well as the compliance and the subjective and objective symptoms of venous outpatients.

\section{Materials and Methods}

\section{Study design}

This study was a prospective, non-interventional/non-invasive observational study conducted between November 2011 (start of recruiting) and February 2015 (end of observational phase) in 47 participating practices in Germany. Decisions regarding diagnosis, treatment, and prescription of mediven MCS were made exclusively by the treating physician, independently of study participation. Mediven MCS (medi $\mathrm{GmbH} \& \mathrm{Co}$. KG) comprise light (mediven elegance, mediven comfort, mediven for men), medium (mediven plus), and firm (mediven forte) MCS characteristics, as well as the mediven ulcer kit, in all variations (standard and made to measure), CCL, and sizes. The term, characteristics' refers to the textile MCS characteristics defining strength, rigidity, and elasticity. All diagnostic measures were performed as recommended $^{3}$ and in accordance with clinical routine practice.

The primary outcomes comprised data
Correspondence: Christine SchwahnSchreiber, Institute of Vascular Medicine, Eschstrasse 10, 21762 Otterndorf, Germany. Tel.: +49.4751.999210 - Fax: +49.4751.999211.

E-mail: schwahn-schreiber@t-online.de

Key words: Medical compression stockings; MCS characteristics; reality of care; CEAP; BMI.

Acknowledgements: we thank Dr. Stefan Lang for his medical editorial assistance with this manuscript. Medical editorial assistance was financed by medi $\mathrm{GmbH} \& \mathrm{Co}$. KG.

Funding: the authors disclosed receipt of the following financial support for the research, authorship, and/or publication of this article: this work was supported by medi $\mathrm{GmbH} \&$ Co. KG (Bayreuth, Germany).

Contributions: CSS, MM, RMS, WD, and SH designed the study. SH and WD was involved in gaining ethical approval and data analysis. All authors were involved in data analysis and interpretation, reviewed and edited the manuscript, and approved the final version.

Conflict of interests: the authors on the scientific advisory board and Ms. Hahn declare that a financial association exists with medi $\mathrm{GmbH}$ \& Co. KG due to an advisory contract. Ms. Doppel is an employee of medi $\mathrm{GmbH} \& \mathrm{Co}$. KG.

Ethical approval: the study was approved by local Ethics Committees and the leading Ethics Committee (State Medical Council of Thuringia, Germany). The study was registered under DRKS00006124 (German Clinical Trials Register).

Note on secondary publication: this article is based on a study first published in 2 articles in Phlebologie [Schwahn-Schreiber C, et al. Long-term observational study on outpatient treatment of venous diseases with medical compression stockings in Germany - The mediven ${ }^{\circledR}$ observational study. Phlebologie 2016;45(1):15-24; Schwahn-Schreiber C, et al. Reality of outpatient care with medical compression stockings. Phlebologie 2016;45(4):207-214].

Received for publication: 15 May 2018

Revision received: 10 August 2018.

Accepted for publication: 10 August 2018.

This work is licensed under a Creative Commons Attribution 4.0 License (by-nc 4.0).

CC Copyright C. Schwahn-Schreiber et al., 2018 Licensee PAGEPress, Italy

Veins and Lymphatics 2018; 7:7560 doi:10.4081/vl.2018.7560 
on the outpatient medical care situation (number and frequency of prescriptions of MCS and donning aids; MCS characteristics), mediven MCS safety and tolerability, and adverse reactions. Secondary outcomes were treatment compliance (frequency and duration of MCS wear) and the subjective and objective venous symptoms of venous outpatients receiving routine care. The study was approved by local Ethics Committees and the leading Ethics Committee (State Medical Council of Thuringia). The study was registered under DRKS0000612419 and performed in compliance with the principles of the Declaration of Helsinki and the mandatory guidelines and regulations.

\section{Inclusion and exclusion criteria}

Male and female patients (CEAP categories C1S - C6) aged between 18 and 79 years with an indication for long-term MCS treatment (18 months) of venous disease (ICD 10) were eligible, if the patient's written informed consent was provided and if patients had not undergone compression treatment during the previous six weeks (including postoperative care with MCS). Patients with a contraindication to MCS, a short life expectancy ( $<2$ years), or a probable need for saphenous vein surgery or flat-knit MCS provision during the observational phase were excluded. Other exclusion criteria were non-venous lymphedema, lipedema, and the absence of physical or mental preconditions required for study participation.

\section{Procedures}

At the beginning of the study, proper fit of the MCS provided was controlled (visit 1). Visit 2 took then place after 1 month, followed by visits 3 to 5 , each in 6-monthsintervals. During the visits, data related to the prescription and provision of MCS and donning aids as well as demographic, physical, and clinical findings were assessed. In addition, frequency and duration of MCS wear, patient-reported MCS tolerability, and the patient's subjective and objective venous symptoms were documented.

\section{Statistics}

Data were collected on a standardized data collection form and documented by the investigator or authorized staff using a web-based system. The authorized clinical research institute (CYCLOMED GmbH) checked all data for consistency, completeness, and plausibility. Statistical analyses were descriptive. For nominal and ordinal-level data, distributions of absolute and relative frequencies are reported. Numerical data are presented as mean \pm standard deviation (SD).

\section{Results}

\section{Patient characteristics}

Out of 841 patients, 784 patients were enrolled. After discontinuation of 253 patients, the population analyzed comprised 531 patients. Patients' characteristics are depicted in Table 1. In brief, $77.6 \%$ of patients were female, and $60.5 \%$ were older than 51 years (Ø $54.2 \pm 14.2$ years $)$. Approximately one third of patients each was of normal weight, overweight, and obese. Most frequent diagnosis was varicose veins of the lower extremities (84.6\%), and most frequent CEAP categories were C2 (34.5\%) and C3 (43.3\%). Frequencies of concomitant diseases and medications were as expected (Table 1).

Representativeness of the population analyzed was confirmed by the association of disease severity, age, and BMI. Elderly patients $>61$ years were more often overweight or obese $(71.2 \% ; n=141)$ than younger patients $<41$ years $(44.9 \%$; $n=40)$, and CEAP class increased with age and BMI. CEAP classes C4 to C6 $(n=53)$ occurred more frequently in patients who were older than 61 years $(67.9 \%)$ and overweight or obese $(83 \%)$.

\section{Outpatient medical care situation}

MCS were of CCL2 (23 - $32 \mathrm{mmHg})$ in almost all prescriptions (98\%; CCL1 [18 - 21 $\mathrm{mmHg}] 1.8 \%$; CCL3 [34 - $46 \mathrm{mmHg}$ ] $0.1 \%$; mediven ulcer kit [2 x $20 \mathrm{mmHg}]$ $0.1 \%)$. The MCS length was mostly AD $(60.2 \%)$, AG $(34.1 \%)$ or AT $(5.7 \%)$, mainly with a closed toe $(60.4 \%$; open: $39.6 \%)$. During the study period, MCS prescriptions

Table 1. Patient characteristics. Population analyzed $\mathbf{N}=531$

\begin{tabular}{lc} 
Sex, $N(\%)$ male / female & $119(22.4) / 412(77.6)$ \\
Age, mean $( \pm$ SD) & $54.2( \pm 14.2)$ \\
\hline Age distribution, N (\%) & \\
$18-20$ & $4(0.8)$ \\
$21-30$ & $39(7.3)$ \\
$31-40$ & $46(8.7)$ \\
$41-50$ & $120(22.6)$ \\
$51-60$ & $123(23.2)$ \\
$61-70$ & $123(23.2)$ \\
$71-79$ & $75(14.1)$ \\
BMI distribution, N (\%) & \\
$<18.5$ & $9(1.7)$ \\
$18.5-24.9$ & $190(35.8)$ \\
$25-29.9$ & $180(33.9)$ \\
$>30$ & $147(27.7)$
\end{tabular}

Frequent diagnoses, $\mathrm{N}(\%) \mathrm{c}$

Varicose veins of lower extremities a) $\quad 449(84.6)$

$\begin{array}{ll}\text { Other venous disorders }{ }^{\text {b) }} & 228(42.9)\end{array}$

$\begin{array}{lc}\text { Clinical classification according to CEAP, N (\%) } & 56(10.6) \\ \text { C1S } & 182(34.5) \\ \text { C2 } & 228(43.3) \\ \text { C3 } & 38(7.2) \\ \text { C4a } & 6(1.1) \\ \text { C4b } & 6(1.1) \\ \text { C5 } & 3(0.6) \\ \text { C6 }\end{array}$

Frequent concomitant diseases, $\mathrm{N}(\%)$ c)

$\begin{array}{ll}\text { Spinal problems } & 202(38)\end{array}$

Hypertension $\quad 175$ (33)

$\begin{array}{ll}\text { Osteoarthritis } & 102(19.2)\end{array}$

Impaired thyroid function $\quad 78$ (14.7)

Diabetes mellitus $\quad 38(7.2)$

$\begin{array}{ll}\text { Frequent concomitant medication, } N(\%) \text { c) } & 94(17.7) \\ \text { Anticoagulants } & 73(13.7) \\ \text { Thyroid hormones } & 73(13.7) \\ \text { Antidepressants } & 67(12.6) \\ \beta \text {-Blockers } & \end{array}$

BMI: body mass index; a) I83.0., I83.1., I83.2., I83.9 (ICD-10-GM-2015); b) I87.0., I87.1., I87.2. I87.9 (ICD-10-GM-2015); c) multiple answers possible; datasets contained missing values (age distribution 0.2\%; BMI distribution 0.9\%; CEAP class 1.5\%). 
were predominantly for light characteristics (light 73.8\%; medium: 23.5\%; firm: $2.1 \%$ ). Importantly, prescriptions of light MCS characteristics dominated irrespective of the patients' CEAP (Figure 1A) and BMI (Figure 1B), and, equally important, this predominance of light characteristics was observed throughout the whole study period.

During the 18-month observational study, patients received an average of $4.1 \pm 1.8$ MCS prescriptions per patient. Approximately one third of patients each received three or less prescriptions during the study $(35.4 \% ; n=188)$, no prescription of a second pair at the beginning of the study $(31.6 \%)$, and no repeated prescription at visit $3(31.3 \%), 4(27.3 \%)$, and 5 $(34.8 \%)$.

Prescriptions of donning aids were generally rare and only slightly increased during the study, from $8.1 \%$ at the beginning $(n=43)$ to $13.8 \% \quad(n=73)$ at visit 5 . Surprisingly, prescriptions of donning aids were not more frequent in cases of obese (11.3\%) and overweight patients (7.1\%) as well as in cases of patients with concomitant diseases such as spinal problems $(9.9 \%)$ and osteoarthritis (11.1\%). Only a higher age resulted in slightly increased prescriptions of donning aids (61-70 years: $12.2 \%$; 71-79 years: $17.3 \%)$.

\section{Safety and tolerability}

There were no major tolerability issues in this study. Leg examinations potentially indicative of impaired MCS tolerability rarely occurred and tended to decrease during the study (Table 2). At the end of the study, constriction furrows at the top band of the MCS, at the popliteal cavity, and at the ankle were found in 17,17 , and 7 patients who wore light $(15,15,6)$ and medium MCS characteristics $(2,2,1)$. No constriction furrows occurred in patients wearing MCS of firm characteristics. Obese or overweight patients who wore MCS of light characteristics had constriction furrows more frequently than those who were underweight or of normal weight.

During the study, between $21.6 \%$ and $29.6 \%$ of patients had dry skin on the leg wearing MCS. At the beginning of the study period, only $36.5 \%$ of participants stated to use skin care products on their legs. Other skin examinations suggesting reduced tolerability were rare, and no relevant skin findings were observed in the majority of patients (Table 2).

\section{Compliance}

At the different study visits, most patients reported to wear the MCS daily (65.3 - 75.6\%) and from morning until evening (48.4-68.4\%). During the study, however, the proportion of patients exhibiting this high level of compliance decreased by $10.3 \%$ (daily) and 20.0\% (morning until evening; Table 3). Compliance to MCS treatment appeared to depend on a number of factors such as the absolute number of MCS prescriptions and the prescription of donning aids. Patients who wore their MCS daily had received more (repeated) prescriptions than patients who wore their MCS occasionally (at study end, the prescriptions per patient ratio was 0.8 for daily users and 0.5 for less frequent users).

A higher number of elderly and obese patients wore their MCS daily or at least on
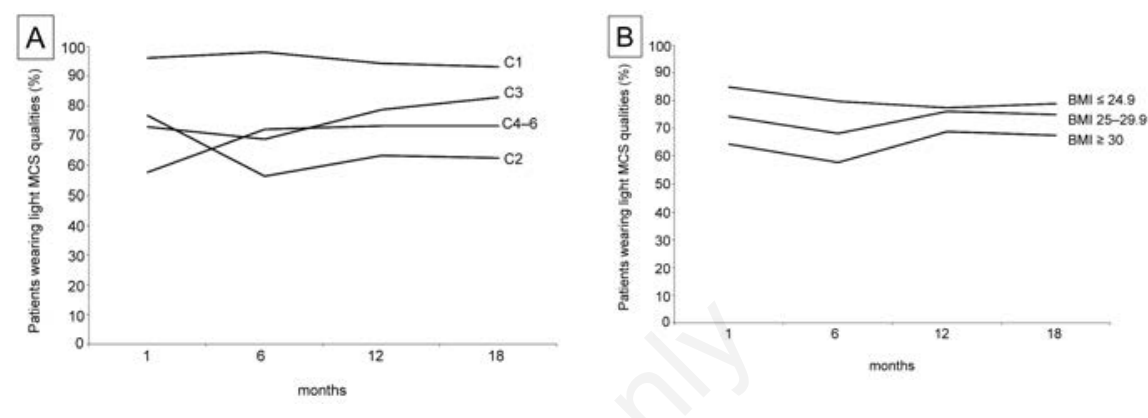

Figure 1. Light characteristics of medical compression stockings (MCS) dominated throughout the study, irrespective of the patients' CEAP (A) and BMI (B). This figure depicts the percentage of light compression stocking characteristics prescribed during the study. The first time point (1 month) includes the screening visit at the start of the study, visit 1 (after 7 days), and visit 2 (after 1 month). Visit 3, 4, and 5 took place after 6, 12, and 18 months. The term characteristics comprises textile MCS features affecting elasticity and other characteristics. Due to the low case numbers, classes $\mathrm{C} 4 \mathrm{a}, \mathrm{C} 4 \mathrm{~b}, \mathrm{C} 5$ and $\mathrm{C} 6$ were pooled. Likewise, weight classes were pooled into BMI $\leq 24.9$, BMI $25-29.9$ and $\mathrm{BMI} \geq 30$.

Table 2. Safety and tolerability of mediven medical compression stockings.

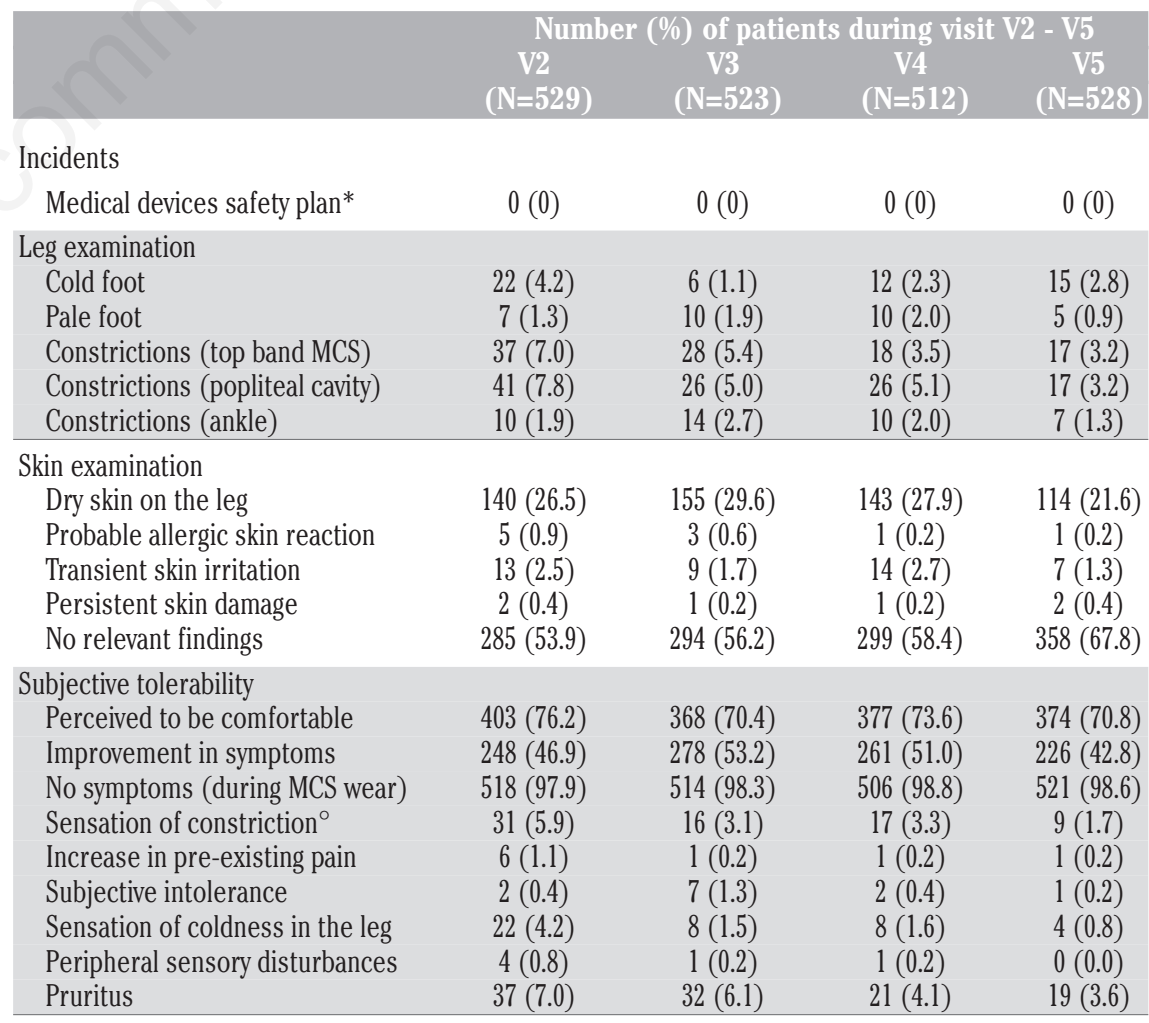

MCS, medical compression stockings. *No incidents, as defined in the Medical Devices Safety Plan Ordinance, occurred; ${ }^{\circ}$ sensation of constriction: instep, in the popliteal cavity, proximal. 
$50 \%$ of days per week (hereafter defined as regular use) if they had received a donning aid prescription. In elderly patients $(\geq 61$ years), the percentage of regular users was higher in the group with a donning aid at both visit 2 and visit 5 (68.4\% and 55.7\%, respectively) than in the group without a donning aid (34.9\% and $34.4 \%$, respectively). Likewise, in obese patients, the percentage of regular users was higher in the group with a donning aid at visit 2 and visit 5 ( $42.1 \%$ and $45.9 \%$, respectively) than in the group without a donning aid $(28.0 \%$ and $27.7 \%$, respectively).

\section{Subjective and objective venous symptoms}

A total of $72 \%$ of patients described the MCS as comfortable. Almost all patients (98\%) reported that wearing the MCS caused no symptoms, and, during the study, subjective venous symptoms improved in almost half of patients (Table 2).

In all CEAP categories, the proportion of patients reporting an improvement of symptoms during the previous 12 months increased by up to $46.1 \%$ whereas the proportion of patients reporting a deterioration decreased by up to $38.7 \%$ during the study.

In contrast to this subjective improvement, a clinically apparent improvement in CEAP class occurred in $17.7 \%$ of patients. In most patients $(72.7 \%)$, the CEAP class remained stable, while deterioration was observed in $9.6 \%$ of patients. In C3 patients $(n=228)$, treatment success can immediately be assessed by edema reduction. A total of $22.4 \%$ of these patients improved during the study (stable: $73.7 \%$; worse $3.5 \%$ ). C3 patients of both normal weight and overweight/obesity benefited more from medium and firm than from light MCS characteristics (Figure 2).

\section{Discussion}

In this observational study, we investigated the provision of MCS and donning aids to venous patients, the safety and tolerability of mediven MCS, the treatment compliance, as well as the subjective and objective symptoms of venous patients in Germany. Although MCS tolerability was very high, and venous symptoms of patients considerably improved during the study, we observed that disease- and patient-specific factors were often insufficiently addressed in MCS prescriptions, potentially affecting compliance and treatment success.

Representativeness of the study population was apparent in the patients' characteristics such as age and BMI, both known to be associated with the prevalence and severity of venous insufficiency. As expected, CEAP class increased with age and BMI, and BMI increased with age. ${ }^{20,21}$ In view of the demographic development and the growing prevalence of overweight and obesity in both developed and developing countries, ${ }^{22}$ our data underline the specific challenges to be considered in the treatment of venous disease in overweight and obese as well as elderly patients.

However, our study revealed that patients' characteristics such as CEAP class, BMI, and age are insufficiently considered when MCS are prescribed in daily practice. In contrast, a uniform standard care prevails. The numbers of MCS and donning aid prescriptions fell well short of what is needed and possible according to health insurance regulations in Germany. Beyond the scheduled six-monthly repeat prescription, more prescriptions would have been possible for medical, hygienic, and safety reasons. ${ }^{3}$ The vast majority of prescribed MCS was of CCL2 and light characteristics, obviously ignoring the patients' CEAP and BMI.
A more frequent prescription of lower as well as higher CCL could have been expected, considering the distribution of patients across all CEAP classes in our study. Likewise, prescriptions of light MCS characteristics predominated throughout the study when compared to those of medium and firm MCS characteristics, even in patients of CEAP classes $>\mathrm{C} 4 \mathrm{a}$ and $\mathrm{BMI}$ $\geq 30$. However, more patients would presumably have required a higher working pressure produced by, for example, MCS of firmer characteristics and corresponding textile characteristics to reduce both venous volume and reflux, and, accordingly, to improve venous pump function. ${ }^{23,24}$ The choice of the textile MCS properties can therefore be a decisive factor for treatment success. ${ }^{25}$ Indeed, improvement in CEAP class was observed more frequently in $\mathrm{C} 3$ patients wearing medium or firm MCS characteristics than in $\mathrm{C} 3$ patients wearing light characteristics. Further studies are now required to establish clearer recommendations on the use of individual MCS characteristics and CCL.

Our study demonstrated a good tolerability of mediven MCS. Approximately half of patients reported an improvement of venous symptoms, up to two thirds of patients stated to perceive the MCS as comfortable, and almost no patient reported on symptoms having occurred during MCS wear. Considering the study duration of 18 months, this result is noteworthy as MCS are often described as comfortable by only less than one third of patients even at shorter study durations. ${ }^{26}$ Adverse events such as constriction furrows were rare and tendentially decreased during the study. Nevertheless, regarding these side effects, we found an association between overweight or obesity and the prescription of light MCS characteristics. Constriction furrows as well as sensation of constriction

Table 3. Compliance to treatment with medical compression stockings (MCS) during the study visits $2-5$.

\begin{tabular}{|c|c|c|c|c|}
\hline & $\begin{array}{c}\text { Numb } \\
\text { V2 } \\
(\mathrm{N}=529)\end{array}$ & $\begin{array}{c}\text { of of patic } \\
\text { V3 } \\
(\mathrm{N}=523)\end{array}$ & $\begin{array}{l}\text { during vis } \\
\text { V4 } \\
(\mathrm{N}=514)\end{array}$ & $\begin{array}{c}\text { it } \begin{array}{c}\mathrm{V}-\mathrm{V} 5 \\
\mathrm{~V} 5 \\
(\mathrm{~N}=527)\end{array} \\
\end{array}$ \\
\hline \multicolumn{5}{|l|}{ Frequency } \\
\hline $\begin{array}{l}\text { Daily } \\
>50 \% \text { of all days in a week } \\
<50 \% \text { of all days in a week } \\
\text { Occasionally } \\
\text { Missing values }\end{array}$ & $\begin{array}{l}400(75.6) \\
91(17.2) \\
25(4.7) \\
10(1.9) \\
3(0.6)\end{array}$ & $\begin{array}{l}364(69.6) \\
94(18.0) \\
34(6.5) \\
28(5.4) \\
3(0.6)\end{array}$ & $\begin{array}{l}365(71.0) \\
82(16.0) \\
30(5.8) \\
32(6.2) \\
5(1.0)\end{array}$ & $\begin{array}{l}344(65.3) \\
110(20.9) \\
26(4.9) \\
42(8.0) \\
5(0.9)\end{array}$ \\
\hline \multicolumn{5}{|l|}{ Daily use } \\
\hline $\begin{array}{l}\text { Morning to evening } \\
\text { Half-days or } 6 \text { - } 8 \text { hours/day } \\
\text { Less than half a day } \\
\text { At and during particular orthostatic stress } \\
\text { Seasonal features } \\
\text { Missing values }\end{array}$ & $\begin{array}{l}362(68.4) \\
139(26.3) \\
10(1.9) \\
6(1.1) \\
5(0.9) \\
7(1.3)\end{array}$ & $\begin{array}{l}304(58.1) \\
154(29.5) \\
9(1.7) \\
16(3.1) \\
38(7.3) \\
2(0.4)\end{array}$ & $\begin{array}{l}291(56.6) \\
171(33.2) \\
4(0.8) \\
24(4.7) \\
16(3.1) \\
8(1.6)\end{array}$ & $\begin{array}{c}255(48.4) \\
197(37.4) \\
4(0.8) \\
21(4.0) \\
42(8.0) \\
8(1.5)\end{array}$ \\
\hline
\end{tabular}

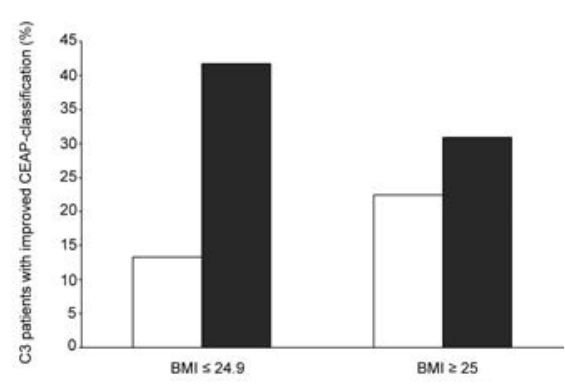

Figure 2. Percentage of C3 patients with improved CEAP class. Patients with BMI $\leq 24.9$ and $\geq 25.0$ had received either light MCS characteristics (white) or medium/firm characteristics (grey). 
predominantly occurred with light characteristics, rarely with medium characteristics, and never with firm characteristics. The occurrence of these side effects therefore additionally emphasizes the importance of both identifying the optimal MCS characteristics for each individual patient and regularly checking the MCS fit.

A considerable number of patients reported on dry skin on the leg wearing MCS. This would have been a preventable symptom, because skin care products were only used by approximately one third of patients. This fact emphasizes the importance of patient education on several aspects of MCS therapy, including both potential side effects and the requirement of regular and long-lasting treatment.

Since chronic venous insufficiency requires a maintenance therapy with daily MCS administration, treatment compliance is essential. Our study confirmed previous publications demonstrating a positive association of the patient's compliance with the number of MCS prescriptions over time ${ }^{27}$ and the prescription of donning aids. ${ }^{28}$ Patients who had received a six-monthly MCS repeat prescription as recommended ${ }^{3}$ as well as elderly and obese patients who had received a donning aid showed a better MCS-wearing frequency, which ultimately improves the prospects for long-term treatment success..$^{29,30}$ Nevertheless, the provision of donning aids did not occur to the extent we would have expected, since donning aids would have been indicated in approximately $25 \%$ of patients. However, in the study relevant relevant comorbidities i. e. spinal problems, osteoarthritis, and obesity did not constitute a relevant indication to prescribe donning aids.

\section{Conclusions}

In conclusion, venous patients are not adequately provided with MCS and donning aids, and prescriptions are generally too undifferentiated regarding $\mathrm{CCL}$ and MCS characteristics to meet the individual needs of patients. This unsatisfactory prescription behavior, which occurred not only at the beginning of the study but also at every visit during the 18-month study period, should be in the focus of future awareness campaigns on compression therapy. Another focus should be on the patient's compliance, especially because it can be easily improved by simple measures such as prescribing a second pair of MCS as well as donning aids. To foster the acceptance of MCS treatment, it is equally important to educate patients on their disease and corre- sponding treatment options as well as on potential side effects. In this context, patients should be informed on the importance of regular skin care. ${ }^{26}$

\section{References}

1. National Clinical Guideline Centre. Varicose veins in the legs: the diagnosis and management of varicose veins. NICE clinical guidelines. London: National Institute for Health and Care Excellence (UK); 2013.

2. Wittens C, Davies AH, Baekgaard N, et al. Editor's choice - management of chronic venous disease: clinical practice guidelines of the European Society for Vascular Surgery (ESVS). Eur J Vasc Endovasc Surg 2015;49:678-737.

3. Wienert V, Gerlach H, Gallenkemper G, et al. Leitlinie: Medizinischer Kompressionsstrumpf (MKS). Phlebologie 2006;35:315-20.

4. Benigni JP, Sadoun S, Allaert FA and Vin F. Efficacy of class 1 elastic compression stockings in the early stages of chronic venous disease. A comparative study. Int Angiol 2003;22:383-92.

5. Cullum N, Nelson EA, Fletcher AW and Sheldon TA. Compression for venous leg ulcers. Cochrane Database Syst Rev 2001:CD000265.

6. Partsch $\mathrm{H}$ and Blattler W. Compression and walking versus bed rest in the treatment of proximal deep venous thrombosis with low molecular weight heparin. J Vasc Surg 2000;32:861-9.

7. Belcaro G, Cesarone MR, Shah SS, et al. Prevention of edema, flight microangiopathy and venous thrombosis in long flights with elastic stockings. A randomized trial: the LONFLIT 4 concorde edema-SSL study. Angiology 2002;53: 635-45.

8. Brandjes DP, Buller HR, Heijboer H, et al. Randomised trial of effect of compression stockings in patients with symptomatic proximal-vein thrombosis. Lancet 1997;349:759-62.

9. Shouler PJ and Runchman PC. Varicose veins: optimum compression after surgery and sclerotherapy. Ann R Coll Surg Engl 1989;71:402-4.

10. Travers JP, Makin GS. Reduction of varicose vein recurrence by use of postoperative compression stockings. Phlebology 1994;4:104-7.

11. Nelson EA and Bell-Syer SE. Compression for preventing recurrence of venous ulcers. Cochrane Database Syst Rev 2012;8:CD002303.

12. Rabe E, Partsch H, Hafner J, et al.
Indications for medical compression stockings in venous and lymphatic disorders: an evidence-based consensus statement. Phlebology 2017;0:1-22.

13. Reich-Schupke S, Murmann F, Altmeyer P, Stücker M. Compression therapy in elderly and overweight patients. Vasa 2012;41:125-31.

14. Raju S, Hollis K, Neglen P. Use of compression stockings in chronic venous disease: patient compliance and efficacy. Ann Vasc Surg 2007;21:790-5.

15. Ziaja D, Kocelak P, Chudek J and Ziaja $\mathrm{K}$. Compliance with compression stockings in patients with chronic venous disorders. Phlebology 2011;26:353-60.

16. Rabe E, Pannier-Fischer F, Bromen K, et al. Bonner Venenstudie der Deutschen Gesellschaft für Phlebologie. Phlebologie 2003;32:1-14.

17. Schwahn-Schreiber C, Marshall M, Murena-Schmidt R, et al. Long-term observational study on outpatient treatment of venous diseases with medical compression stockings in Germany: The mediven ${ }^{\circledR}$ observational study. Phlebologie 2016;45:15-24.

18. Schwahn-Schreiber C, Marshall M, Murena-Schmidt R, et al. Reality of outpatient care with medical compression stockings. Phlebologie 2016;45: 207-14.

19. German Clinical Trials Register. A noninterventional study of mediven ${ }^{\circledR}$ compression stockings on the question of product safety and compliance in patients with phlebological disease in outpatient care according to $\$ 23 \mathrm{~b}$ german medical devices act; 2014. Available from: https://drks-neu.uniklinik-freiburg.de/drks_web/ setLocale EN.do) Accessed: 21 May 2017.

20. Rabe E, Guex JJ, Puskas A, et al. Epidemiology of chronic venous disorders in geographically diverse populations: results from the vein consult program. Int Angiol 2012;31:105-15.

21. Reich-Schupke S. Compression therapy in obese patients. Phlebologie 2015; 44:71-6.

22. Ng M, Fleming $\mathrm{T}$, Robinson $\mathrm{M}$, et al. Global, regional, and national prevalence of overweight and obesity in children and adults during 1980-2013: a systematic analysis for the Global Burden of Disease Study 2013. Lancet 2014;384:766-81.

23. Mosti G. Relevance of stiffness of compression material on venous hemodynamics and edema. Veins and Lymphatics 2013:26-9.

24. Partsch H, Schuren J, Mosti G and Benigni JP. The static stiffness index: an important parameter to characterise 
compression therapy in vivo. J Wound Care 2016;25 Suppl 9:S4-S10.

25. Partsch H, Partsch B, Braun W. Interface pressure and stiffness of ready made compression stockings: comparison of in vivo and in vitro measurements. J Vasc Surg 2006;44:809-14.

26. Reich-Schupke S, Murmann F, Altmeyer P, Stücker M. Quality of life and patients' view of compression therapy. Int Angiol 2009;28:385-93.
27. Institut für Demoskopie Allensbach. Mehr Lebensqualität, weniger Schmerzen: Nutzung und Wirksamkeit orthopädischer Hilfsmittel. eurocom e. V. 2015:1-25.

28. Sippel K, Seifert B, Hafner J. Donning devices (foot slips and frames) enable elderly people with severe chronic venous insufficiency to put on compression stockings. Eur J Vasc Endovasc Surg 2015;49:221-9.
29. Kostas TI, Ioannou CV, Drygiannakis I, et al. Chronic venous disease progression and modification of predisposing factors. J Vasc Surg 2010;51:900-7.

30. Rastel D. Treatment by medical compression stockings among 144 consecutive patients with non-complicated primary varicose veins: results on compliance. J Mal Vasc 2014;39:389-93. 